\title{
Optical vortices and Airy' spiral in chiral crystals
}

\author{
Volyar A., Rubass A., Shvedov V., Fadeyeva T. and Kotlyarov K. \\ Physics Department, Taurida National V. Vemadsky University, 4 Vernadsky \\ Ave., 95007 Simferopol, Crimea, Ukraine, e-mail: volyar@crimea.edu
}

Received: 20.06.2007

After revision: 20.08.2007

\begin{abstract}
We consider both theoretically and experimentally propagation of a singular beam along a single chiral crystal and a stack of two such crystals with the opposite signs of their chirality coefficients. We develop a matrix approach for describing behavior of the beam singularities. The beams with the eigen polarization turn out to carry centered optical vortices with double topological charges. At the same time, a circularly polarized beam, when propagating, acquires an additional unrequited phase having much to do with the geometrical Pancharatnam phase. The sign of the phase is defined by a direction of polarization circularity. To display experimentally the distribution of the geometrical phase we suggest employing two circularly polarized beams with opposite the circularities. The spiral image appearing behind the binary crystal system and the polarization filter represents a set of spiral and ring edge dislocations of the wave front. They outline a profile of geometrical phase of the beam. Such the dislocation system is known in optical crystallography. It is nothing else than the four-fold Airy' spiral. We show also that the system of a chiral crystal with a purely anisotropic crystal, as well as a single chiral crystal combined with a circular polarization filter, are able to form a dark spiral line, too. However, such the line does not represent the edge dislocation and is shaped by means of a chain of optical vortices.
\end{abstract}

Keywords: optical vortex, geometrical phase, Airy' spiral

PACS: 41.85.-p, 42.25.Ja, 42.25.Lc

\section{Introduction}

Birefringent crystals represent promising media for producing vortex-bearing beams $[1$, 2]. Among a variety of vortex-beam properties in anisotropic optically uniaxial crystals (see, e.g., [3-15]), one can single out appearance of a degenerate singular point (a socalled $C$ point [16]) at the beam axis, which is encircled by contours with sharply shifting polarization states. In terms of scalar waves, such a singular point corresponds to a doubly charged optical vortex $[4-7,11,14]$, i.e. a circularly polarized components of the beam propagating along a crystal axis carry optical vortices with the Gaussian envelope [17]. Moreover, such crystals have enabled us to produce high-quality polychromatic optical vortices with a double topological charge and high energy efficiency $[4,6,11,19-$ 
21]. Notice that a conical refraction in biaxial crystals not only promotes generation of Bessel-Gaussian beams $[3,10,12]$ but also produces singly charged optical vortices $[8$, $13,14]$.

Beside of chirality (optical activity) caused by a circular birefringence, a majority of available optically transparent crystals have also a linear birefringence, resulting in splintering a wave field into ordinary and extraordinary beams. Such chiral crystals transform simultaneously the polarization state and evoke additional rotation of the polarization ellipse at each point of beam cross-section [22]. Availability of chirality in crystals complicates essentially the process of formation of singular beams $[9,18]$, changing a fine structure of optical vortices via deformation of beam fields with eigen polarizations. Indeed, a plane wave transmitting at some angle to the optic axis of a purely anisotropic crystal has two linear eigen polarizations directed along and perpendicular to the plane formed by the wave normal and the crystal optic axis [23]. However, eigen polarizations of plane waves in chiral crystals are elliptical ones, their polarization state depending on the wave propagation direction $[24,25]$. Thus, a beam with the eigen polarization acquires a complex distribution of polarization states over its cross-section. It cannot help provoking generation of new features of singular points in vector fields.

The aim of this work is to study theoretically and experimentally polarization and phase singularities of the beam field for a system of chiral crystals.

The paper includes four sections. The matrix approach for describing beam propagation through a chiral crystal is considered in the first section. The second section is devoted to analysis of field structure of the beams with eigen polarization. The third section touches upon a question of birth and annihilation processes of optical vortices in topologically neutral beams transmitting through a system of two chiral crystals and a polarization filter. We show there that the beam field after the system acquires a geometrical phase. We discuss also the experiment on detecting this excess phase. In the fourth section we display that the well-known Airy' spiral is a result of superposition of two vortex beams, whose fields are perturbed by geometrical phases with the opposite signs. Finally, in the Appendix we consider geometrical phase properties and derive the condition for the Airy' spiral.

Let us build a model for the Gaussian beam propagation along the optic axis of a uniaxial chiral crystal. Let the optic axis of crystal is directed along $\zeta$ axis of a lab frame of reference. We assume for simplicity that the optical activity is the same for all crystal directions. The permittivity tensor $\hat{\boldsymbol{\varepsilon}}$ for the plane wave with the wave vector $\mathbf{k}=\left\{\begin{array}{lll}k_{x} & k_{y} & k_{z}\end{array}\right\}$ can be written as [22]

$$
\varepsilon_{i k}=\varepsilon_{i k}^{(0)}+i e_{i k l} g_{l m} k_{m},
$$

where $e_{i k l}$ is the antisymmetric unit tensor, $\mathrm{g}_{\mathrm{lm}}$ stands for the gyration tensor. The tensor $\varepsilon_{i k}^{(0)}$ describes the linear birefringence of the crystal. 
The diagonal elements $\varepsilon_{11}=\varepsilon_{22}=\varepsilon_{o}$ and $\varepsilon_{33}=\varepsilon_{e}$ of the first tensor $\varepsilon_{i k}^{(0)}$ in Eq.(1) enable us to find a permittivity for the waves transmitting along the $\mathbf{k}$ direction [23] so that:

$$
\frac{1}{\varepsilon^{\prime \prime}}=\frac{\cos ^{2} \theta}{\varepsilon_{o}}+\frac{\sin ^{2} \theta}{\varepsilon_{e}},
$$

the angle $\theta$ is formed by the wave vector $\mathbf{k}$ and the crystal optical axis (see Fig.1a,c)

The theoretical analysis of the beam propagation in a pure anisotropic crystal (a crystal with a linear birefringence only) in the paraxial approximation can be done with either the method of spectral integral [7] or the method of mode beams [17]. In our case of chiral crystal, it is also possible to employ both methods (see e.g. Ref. [9, 19]) though, for simplicity we will make use of an approximate matrix approach similar to that presented in Refs. [4, 6, 17].

First of all, we note that paraxial vortex beams in a uniform medium can be described in terms of plane waves with the wave vectors lying on the surface of hyperboloid of revolution [26-28] (see Fig. la). In the case of purely anisotropic crystal we can work

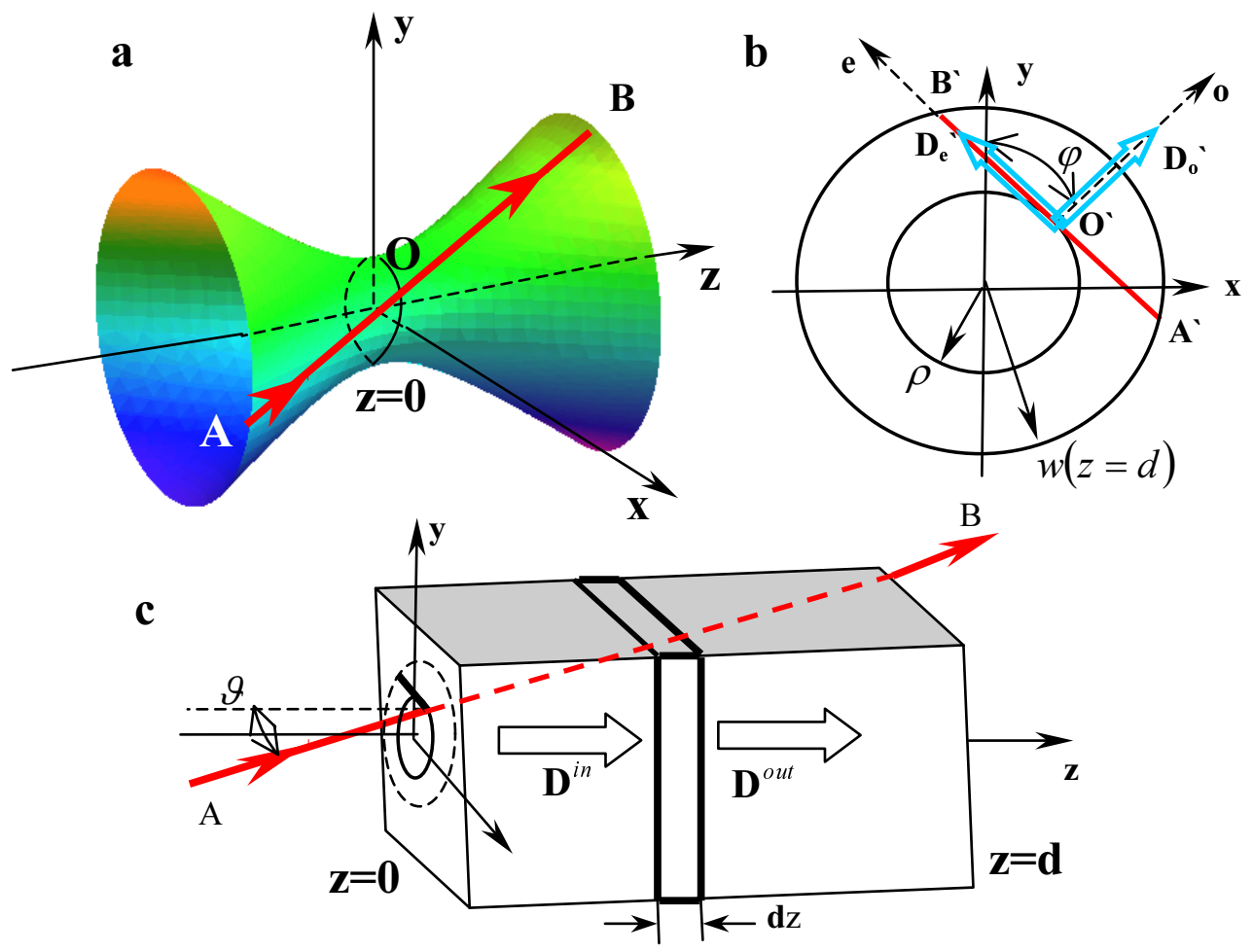

Fig. 1. The sketch of the beam propagation in chiral crystals: (a) the ray $A B$ in the beam laying on the surface of hyperboloid of revolution, (b) the ray projection onto the beam cross-section, and (c) transformation of the induction vector $\mathbf{D}$. The optic axis of crystal is directed along the $z$ axis. 
in a similar way $[6,7]$. In our particular case, we assume that the crystal chirality is very small, i.e. $\Delta n_{C}<\Delta n_{L}\left(\Delta n_{L}=n_{o}-n_{e}\right)$ so that the method mentioned above can be used, too.

Let us consider the wave normal trajectory AOB (see Fig. la) lying on the hyperboloid surface. The wave vector $\mathbf{k}$ of the electric induction $\mathbf{D}$ is directed along the normal to the wave front, while the vectors with eigen polarizations lay in the plane perpendicular to the wave vector. We will seek for the transformation matrix $\hat{\mathbf{M}}$ for the electric induction $\mathbf{D}$ of the Gaussian beam propagating in the crystal. Let us divide the crystal into $N$ elements with the thickness $\Delta z$ (see Fig. lc) and assume that, at first, the crystal changes the polarization state of the wave via the linear birefringence and then rotates its polarization plane via the circular birefringence. Each section of the crystal induces the phase difference between the $\mathbf{D}$ components, which in the linear basis is equal to $\Delta_{N}=\frac{\delta}{N}$, where (see [23])

$$
\delta \approx k \Delta n_{L} L \sin ^{2} \theta \approx k z \Delta n_{L} k_{\perp}^{2}
$$

where $L$ is the geometrical length $\mathrm{AOB}$ ( $L \rightarrow z$ in the paraxial approximation and $z=d$ is the crystal thickness), $k_{\perp}^{2}=\sqrt{k_{x}^{2}+k_{y}^{2}}$ stands for the transverse wave number. The first transformation matrix has the form (see, e.g., Ref [27])

$$
\hat{\mathbf{G}}=\left(\begin{array}{cc}
\cos \frac{\delta}{2 N}+i \sin \frac{\delta}{2 N} \cos 2 \varphi & i \sin \frac{\delta}{2 N} \sin 2 \varphi \\
i \sin \frac{\delta}{2 N} \sin 2 \varphi & \cos \frac{\delta}{2 N}-i \sin \frac{\delta}{2 N} \cos 2 \varphi
\end{array}\right) .
$$

On the other hand, the action of the circular birefringence can be presented in the same basis as

$$
\hat{\mathbf{g}}=\left(\begin{array}{cc}
\cos \frac{\gamma}{N} & -\sin \frac{\gamma}{N} \\
\sin \frac{\gamma}{N} & \cos \frac{\gamma}{N}
\end{array}\right)
$$

where $\gamma=\Delta n_{C} d \sqrt{k_{\perp}^{2}+k^{2}}$. If the element thickness is very small $\Delta z \rightarrow 0$ then the matrices (4) and (5) commute and we have

$$
\hat{\Gamma}_{N}=\hat{g} \hat{G}=\left(\begin{array}{cc}
1+i \frac{\delta}{2 N} \cos 2 \varphi & i \frac{\delta}{2 N} \sin 2 \varphi \\
i \frac{\delta}{2 N} \sin 2 \varphi & 1-i \frac{\delta}{2 N} \cos 2 \varphi
\end{array}\right)\left(\begin{array}{cc}
1 & -\frac{\gamma}{N} \\
\frac{\gamma}{N} & 1
\end{array}\right) \approx \hat{1}+\hat{\Delta}_{N}+\hat{\gamma}_{N},
$$

where

$$
\hat{\Delta}_{N}=i \frac{\delta}{2 N}\left(\begin{array}{cc}
\cos 2 \varphi & \sin 2 \varphi \\
\sin 2 \varphi & -\cos 2 \varphi
\end{array}\right), \quad \gamma=\frac{\gamma}{N}\left(\begin{array}{cc}
0 & -1 \\
1 & 0
\end{array}\right)
$$

The transformation matrix of the crystal can be written as 


$$
\hat{\mathbf{M}}=\lim _{N \rightarrow \infty} \Pi\left(1+\hat{\Delta}_{N}+\hat{\gamma}_{N}\right) \approx \lim _{N \rightarrow \infty} \prod_{N} \exp \left(\hat{\Delta}_{N}+\hat{\gamma}_{N}\right)=\exp \left(\int_{0}^{d}[\hat{\Delta}(z)+\hat{\gamma}(z)] d z\right)
$$

This may be reduced to the standard form [29]:

$$
\hat{\mathbf{M}}=\left(\begin{array}{cc}
\cos \Lambda+i \frac{\delta}{2 \Lambda} \sin \Lambda \cos 2 \varphi & \frac{\gamma+i \frac{\delta}{2} \sin 2 \varphi}{\Lambda} \sin \Lambda \\
\frac{-\gamma+i \frac{\delta}{2} \sin 2 \varphi}{\Lambda} \sin \Lambda & \cos \Lambda-i \frac{\delta}{2 \Lambda} \sin \Lambda \cos 2 \varphi
\end{array}\right)
$$

where $\Lambda=\sqrt{(\delta / 2)^{2}+\gamma^{2}}$.

The matrix (9) transforms the transverse components of the induction vector according to

$$
\left(\begin{array}{c}
D_{x} \\
D_{y}
\end{array}\right)^{\text {out }}=\hat{\mathbf{M}}\left(\begin{array}{c}
D_{x} \\
D_{y}
\end{array}\right)^{\text {in }}
$$

If the field distribution at the plane $z=0$ corresponds to Gaussian envelope,

$$
\Psi(z=0)=\exp \left(-\frac{r^{2}}{\rho^{2}}\right),
$$

where $\rho$ is the waist radius, then its Fourier transform of the wave vector $\mathbf{k}$ distribution is as follows:

$$
\Psi\left(k_{x}, k_{y}\right)=\exp \left(-\frac{\rho^{2} k_{\perp}^{2}}{2}\right)
$$

The spectral integral for the field at the arbitrary plane $\mathrm{z}$ of crystal in the paraxial approximation is

$$
\Psi_{\perp}\left(r, \varphi^{\prime}, z\right)=\int_{0}^{2 \pi} d \varphi \int_{0}^{\infty} k_{\perp} d k_{\perp} \hat{\mathbf{M}} \mathbf{D}^{i n} \Psi\left(k_{\perp}\right) \exp \left\{i k_{\perp} r \cos \left(\varphi-\varphi^{\prime}\right)+i k_{z} z\right\}
$$

where $k_{z}=\sqrt{k^{2}-k_{\perp}^{2}}$. The major contribution to the integral (11) is given by the directions of the wave vector $\mathbf{k}$ lying on the surface of hyperboloid of revolution. Thus, we have

$$
\Psi_{\perp}\left(r, \varphi^{\prime}, z=d\right) \approx \hat{\mathbf{M}}_{L} \mathbf{D}^{i n} \Psi(r)
$$

where

$$
\hat{\mathbf{M}}_{L}=\left(\begin{array}{cc}
\cos \Lambda^{\prime}+i \cos \alpha \sin \Lambda^{\prime} \cos 2 \varphi^{\prime} & \left(\sin \alpha+i \cos \alpha \sin 2 \varphi^{\prime}\right) \sin \Lambda^{\prime} \\
\left(-\sin \alpha+i \cos \alpha \sin 2 \varphi^{\prime}\right) \sin \Lambda^{\prime} & \cos \Lambda^{\prime}-i \cos \alpha \sin \Lambda^{\prime} \cos 2 \varphi^{\prime}
\end{array}\right)
$$

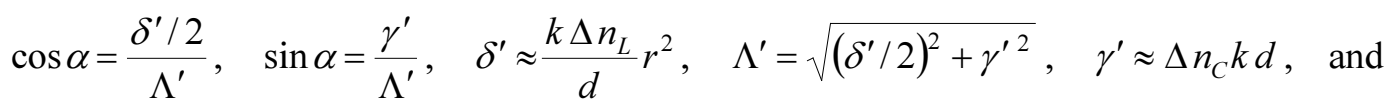
$k=\sqrt{\varepsilon} k_{0}$, here $\varphi^{\prime}$ is the azimuthal coordinate of the observation point at the beam 
cross-section and

$$
\Psi(r, z=d)=\exp \left(-i k \frac{r^{2}}{2 Z}\right) / Z \exp (i k d), Z=d+i z_{0}, z_{0}=\frac{k \rho^{2}}{2} .
$$

Since the wave vectors for low-order Laguerre-Gaussian beams bearing optical vortices are also required to lye on the hyperboloid surface, we can rewrite the function (12) as

$$
\Psi(x, y, z=d)=\left(\frac{x \pm i y}{Z}\right)^{|l|} \exp \left(-i k \frac{r^{2}}{2 Z}\right) / Z \exp (i k d),
$$

where $l$ is the topological vortex charge at the plane $z=0$. The above requirement is not fulfilled for high-order Laguerre-Gaussian beams so that we cannot use the transformation (12) in these cases.

\section{Singular beams with the eigen polarization}

Let us consider behavior of the beams transmitting along the $\mathrm{z}$ axis (consequently, along the optic axis) in the chiral crystal, which has the eigen polarization of the beams in purely birefringent crystal. It is well-known $[7,17]$ that the singular beams with the eigen polarization of the lowest order represent $|T E\rangle$ and $T M\rangle$ modes:

$$
\left.T E\rangle=\left(\begin{array}{c}
y \\
-x
\end{array}\right) \Psi(r, z) / Z, \quad T M\right\rangle=\left(\begin{array}{l}
x \\
y
\end{array}\right) \Psi(r, z) / Z .
$$

Using Eqs. (12), (13) and (16), we can plot polarization distribution of the field after the crystal. Fig. 2 is a result of the corresponding computer simulations. It is seen that the
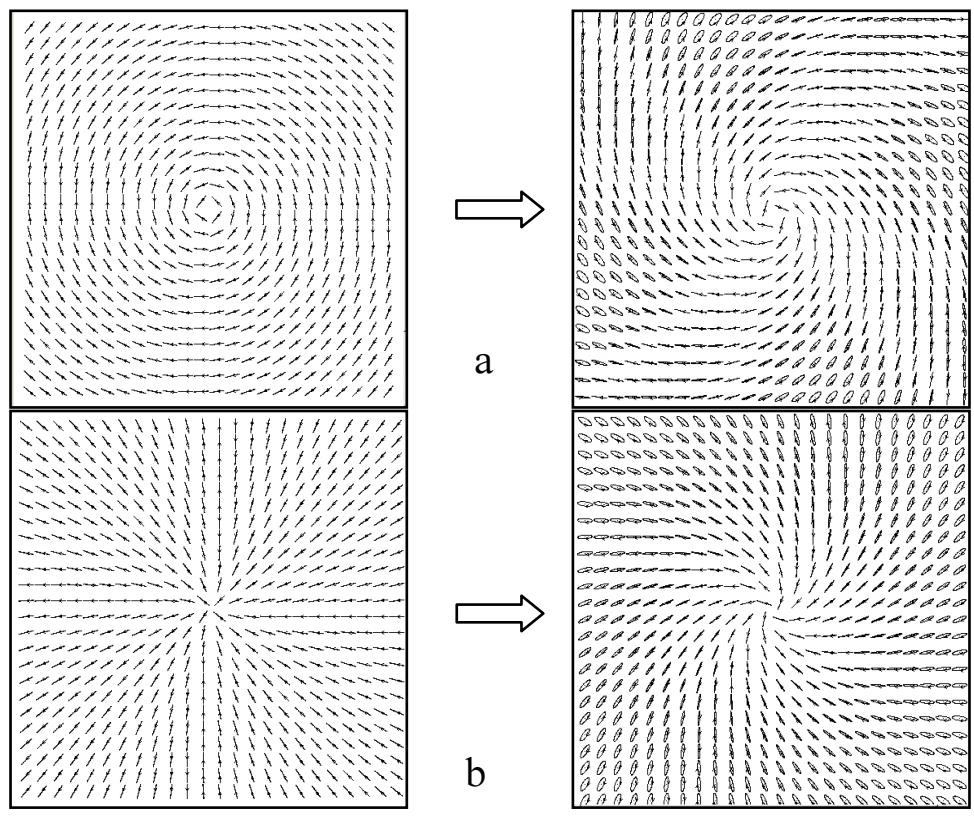

Fig. 2. Transformation of mode beams with the TE and TM polarizations in the chiral crystal with $\Delta n_{L}=2 \cdot 10^{-3}, \Delta n_{C}=3 \cdot 10^{-5}$ and $d=1 \mathrm{~cm}$ : (a) TE mode and (b) TM mode. 
field structure changes essentially and the straight lines turn into spiral ones. However, in contrast to Ref [30] that describes the evolution of singular vector beams with a spiral orientation of linear polarization, here we deal with the elliptical polarizations. The spiral lines in our case are envelopes to a set of major ellipse axes. With moving the observation point off the centre, the ellipse eccentricity gets larger.

To find polarization distribution of the beams with the eigen polarization in chiral crystals, it is necessary at first to write the matrix (13) in the circularly polarized basis $\hat{\mathbf{M}}_{C}$. Here we make use of the transition matrices:

$$
\hat{\mathbf{L}}=\left(\begin{array}{cc}
1 & -i \\
1 & i
\end{array}\right), \quad \hat{\mathbf{C}}=\frac{1}{2}\left(\begin{array}{cc}
1 & 1 \\
i & -i
\end{array}\right) .
$$

Then $\hat{\mathbf{M}}_{C}=\hat{\mathbf{L}} \hat{\mathbf{M}}_{L} \hat{\mathbf{C}}$ or

$$
\hat{\mathbf{M}}_{C}=\frac{1}{2}\left(\begin{array}{cc}
\cos \Lambda^{\prime}-i \sin \alpha \sin \Lambda^{\prime} & i \cos \alpha \sin \Lambda^{\prime} \exp \left(-i 2 \varphi^{\prime}\right) \\
i \cos \alpha \sin \Lambda^{\prime} \exp \left(i 2 \varphi^{\prime}\right) & \cos \Lambda^{\prime}+i \sin \alpha \sin \Lambda^{\prime}
\end{array}\right) .
$$

The eigen values of the matrix (17) are

$$
h=\exp ( \pm i \Lambda),
$$

while its eigen vectors in the circularly polarized basis are represented by

$$
\mathbf{m}^{+}=\left(\begin{array}{c}
\cos \alpha \exp \left(-i \varphi^{\prime}\right) \\
(1+\sin \alpha) \exp \left(i \varphi^{\prime}\right)
\end{array}\right), \quad \mathbf{m}^{+}=\left(\begin{array}{c}
(1+\sin \alpha) \exp \left(-i \varphi^{\prime}\right) \\
-\cos \alpha \exp \left(i \varphi^{\prime}\right)
\end{array}\right) .
$$

By using the method of Stokes parameters [23, 31], we come to the expressions for the inclination angle of polarization ellipse $\psi$,

$$
\tan 2 \psi=\tan 2 \varphi
$$

as well as the ellipticity as the ellipse axes ratio:

$$
Q=\frac{1}{2} \sec \frac{\alpha}{2}
$$

Fig. 3 illustrates the maps of polarization distributions corresponding to the eigen-

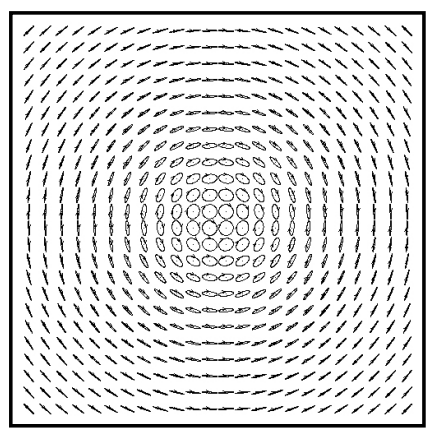

a

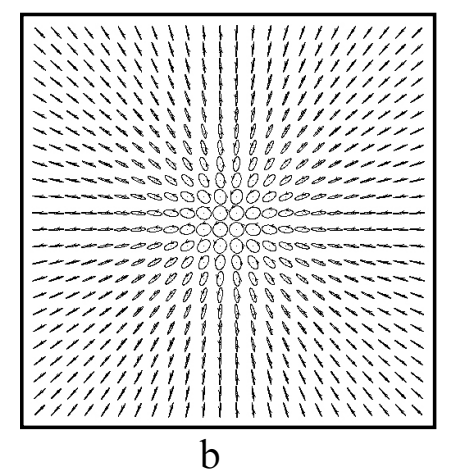

Fig. 3. Polarization distributions in the beams $\mathbf{m}^{+}$(a) and $\mathbf{m}^{-}$(b): $\Delta n_{L}=2 \cdot 10^{-3}$, $\Delta n_{C}=3 \cdot 10^{-5}$ and $d=2 \mathrm{~cm}$. 
vectors given by Eqs. (18). The circular polarization is positioned at the beam axis. This is the so-called $C$ points [16]. As the observation point moves off the beam axis, the ellipse eccentricity decreases and the polarization tends to linear one. The integral curves for the vector $\mathbf{m}^{+}$represent a set of concentric circles with the centre located at the axis. The integral curves for the vector $\mathbf{m}^{-}$are the rays with a common centre lying at the axis. In fact, the beams with the eigen polarization carry a doubly charged optical vortex nested in one of the field components. While the beam transmits along the chiral crystal, the field structure does not change with the accuracy of a scale factor.

\section{Optical vortex generation in single and binary chiral crystals 3.1. Single crystal}

Let us analyze the field structure for the beam passed through a uniaxial chiral crystal. provided that the initial Gaussian beam transmitting along the optic axis is circularly polarized at the plane $z=0$ :

$$
\Psi^{i n}(z=0)=\left(\begin{array}{c}
D^{+} \\
D^{-}
\end{array}\right) \Psi(r)=\left(\begin{array}{l}
1 \\
0
\end{array}\right) \Psi(r)
$$

where the function $\Psi(r)$ is described by Eq. (10a). Using Eqs. (12) and (19), we come to

$$
\boldsymbol{\Psi}^{\text {out }}=\hat{\mathbf{M}}_{C} \boldsymbol{\Psi}^{i n}=\frac{1}{2}\left(\begin{array}{c}
\cos \Lambda^{\prime}-i \sin \alpha \sin \Lambda^{\prime} \\
i \cos \alpha \sin \Lambda^{\prime} \exp \left(-i 2 \varphi^{\prime}\right)
\end{array}\right) \Psi(r) .
$$

The maps of the field distributions plotted in accordance with Eq. (20) are shown in Fig. 4. The $C$ point is positioned at the pattern centre. Around it, major axes of the polarization ellipses form a regular spiral-like set of curves. In contrast to a similar pattern of the field appearing after purely anisotropic crystal [6], in the chiral crystal we observe a more extensive portion of the field with nearly circular polarization in the vicinity of the centre, while the integral lines are sharply twisted outside it. The senses of the torsion are opposite for the left and right circular polarizations of the initial beam.

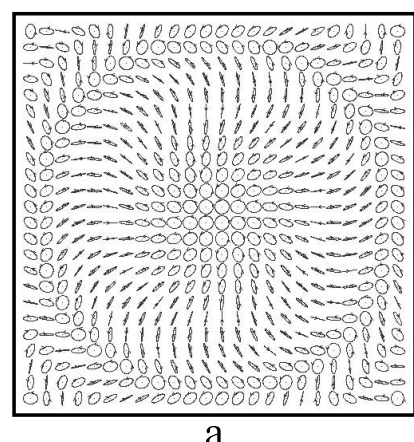

a

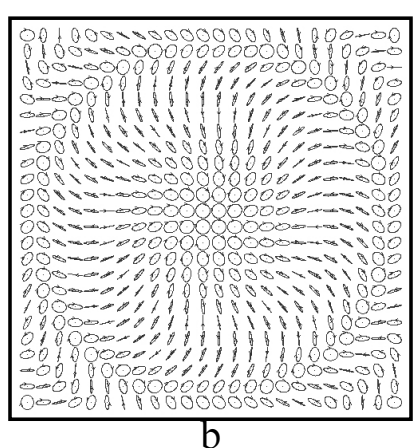

b

Fig. 4. The map of the beam field structure with the initial right (a) and left (b) circular polarizations: $\Delta n_{L}=0.02, \Delta n_{C}=1.5 \cdot 10^{-5}$ and $d=1 \mathrm{~cm}$. 


\subsection{Binary chiral crystal}

Let us try to compensate the polarization plane rotation with employing a second chiral crystal with the opposite sign of the chirality. Naturally, such a binary system cannot compensate the crystal chirality completely. However, it does underline concealed, inherent properties of crystal-generated optical vortices.

One can obtain the matrix of the binary system by multiplying the matrices (17) with the opposite signs of the parameter $\gamma$ :

$$
\hat{\mathbf{M}}_{C}^{(2)}=\frac{1}{4}\left(\begin{array}{cc}
\cos ^{2} \Lambda^{\prime}-\cos 2 \alpha \sin ^{2} \Lambda^{\prime} & i 2 \cos \alpha \sin \Lambda^{\prime}\left(\cos \Lambda^{\prime}-i \sin \alpha \sin \Lambda^{\prime}\right) e^{-i 2 \phi^{\prime}} \\
i 2 \cos \alpha \sin \Lambda^{\prime}\left(\cos \Lambda^{\prime}+i \sin \alpha \sin \Lambda^{\prime}\right) e^{i 2 \varphi^{\prime}} & \cos ^{2} \Lambda^{\prime}-\cos 2 \alpha \sin ^{2} \Lambda^{\prime}
\end{array}\right) .
$$

When the initial Gaussian beam $\left(\begin{array}{l}1 \\ 0\end{array}\right) \Psi(r)$ propagates, the wave field is described by the expression

$$
\boldsymbol{\Psi}^{\text {out }}=\left(\begin{array}{c}
D^{+} \\
D^{-}
\end{array}\right)=\frac{\Psi(r, z)}{4}\left(\begin{array}{c}
\cos ^{2} \Lambda^{\prime}-\cos 2 \alpha \sin ^{2} \Lambda^{\prime} \\
i 2 \cos \alpha \sin \Lambda^{\prime}\left(\cos \Lambda^{\prime}+i \sin \alpha \sin \Lambda^{\prime}\right) e^{i 2 \varphi^{\prime}}
\end{array}\right),
$$

As a result, the $D^{-}$component carries a doubly charged optical vortex. In the Appendix, we show that this vortex beam acquires a phase access that has much to do with the Pancharatnam phase $[32,33]$. This phase is a peculiar indicator of the beam state. So if the Pancharatnam phase is not zero, the vector field can be structurally unstable and it bears an orbital angular momentum [33]. The beam with the opposite circular polarization $\left(\begin{array}{l}0 \\ 1\end{array}\right) \Psi(r)$ acquires the geometrical phase with the opposite sign. The phase access affects the vortex structure. Indeed, Fig. 5 demonstrates the wave front surface for the $D^{-}$beam component. While the wave front of the beam transmitted through a single chiral crystal has a form of straight helicoid (Fig. 5b), the form of that helicoid is deformed after the binary crystal. The helicoid generatrix then becomes spiral (see Fig. 5a).

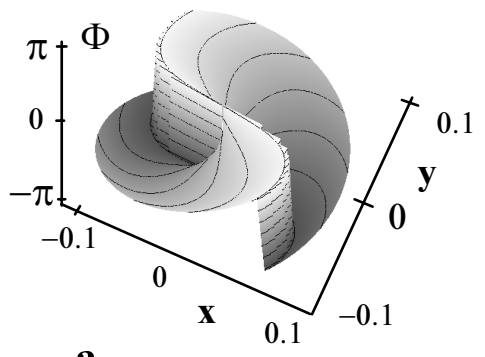

a

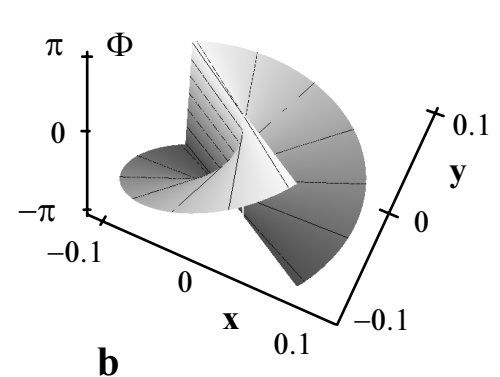

b

Fig. 5. The axial portion of the $D^{-}$component wave front after the binary crystal (a) and the single crystal (b). 


\section{Airy' spirals, optical vortices and the geometrical phase}

Generally speaking, spiral-like equiphase lines are intrinsic to any free-propagating vortex beam because of its spatial divergence. As a result, we observe a spiral pattern in the interference experiment $[34,35]$. Thus, it is necessary to use a more accurate experimental approach for bringing to light the phase access. A necessary condition for detecting the vortex geometrical phase is careful control of the wave front form for two superposed beams. It could be achieved if the two beams are subjected to wave transformations, under which their dynamic phases change identically while the geometrical phases experience different perturbations. The combined action of two crystals and a linear polarizer results in a characteristic pattern of the intensity distribution shown in Fig.6.

In polarization optics, the images presented in Fig. 6 have come to be called conoscopic patterns $[22,25]$, whereas the spiral-like or fylfot-like conoscopic patterns are called as fourfold Airy 'spirals [36,37].

Let us analyze the physical nature of these spirals. To this end we write the matrix (21) in the linearly polarized basis, using the relation $\hat{\mathbf{M}}_{C}=\hat{\mathbf{C}} \hat{\mathbf{M}}_{L} \hat{\mathbf{L}}$ and Eq. (16a), so that we come finally to the equation

$$
\mathbf{D}^{(i n)}=\left(\begin{array}{l}
D_{x} \\
D_{y}
\end{array}\right)=\left(\begin{array}{l}
1 \\
0
\end{array}\right) \Psi(r)
$$

where

$$
\begin{gathered}
D_{x}=\left[\cos ^{2} \Lambda^{\prime}-\cos 2 \alpha \sin ^{2} \Lambda^{\prime}+i \cos \alpha \sin \Lambda^{\prime}\left(\cos \Lambda^{\prime} \cos 2 \varphi^{\prime}+\sin \alpha \sin \Lambda^{\prime} \sin 2 \varphi^{\prime}\right)\right] \Psi(r), \\
D_{y}=i 2 \cos \alpha \sin \Lambda^{\prime}\left(\cos \Lambda^{\prime} \sin 2 \varphi^{\prime}-\sin \alpha \sin \Lambda^{\prime} \cos 2 \varphi^{\prime}\right) \Psi(r)
\end{gathered}
$$
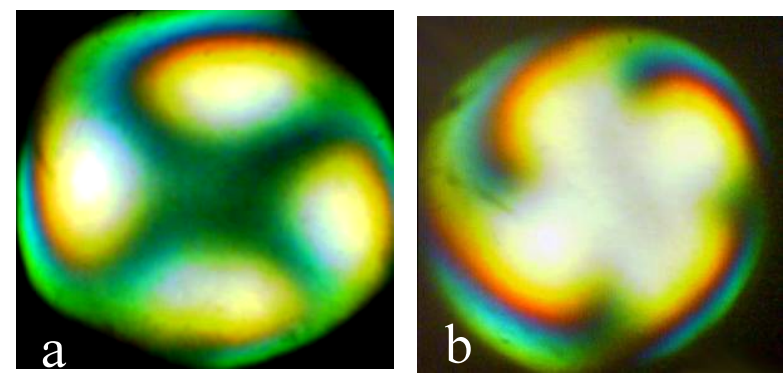

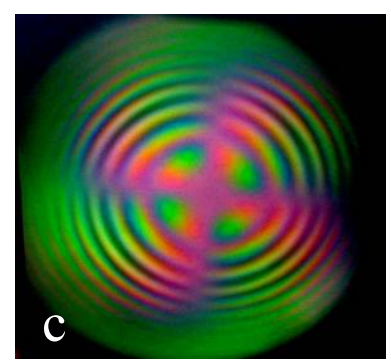

$\beta=\pi / 2$

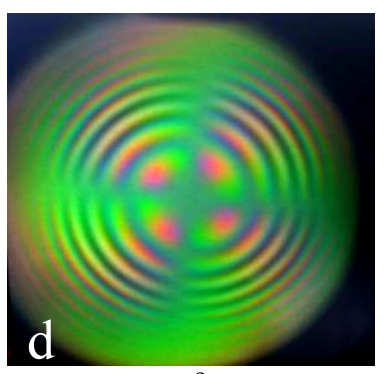

$\beta=0^{\circ}$

Fig. 6. Intensity distribution for the beam passed through the binary crystal system and the polarizer: $(a, b)$ two chiral crystals; $(c, d)$ chiral crystal and purely anisotropic crystal. 
From Eq. (23) we find that the $D_{y}$ component vanishes if

$$
\begin{aligned}
& \cos \Lambda^{\prime} \sin 2 \varphi+\sin \Lambda^{\prime} \cos 2 \varphi=0, \\
& \sin \Lambda^{\prime}\left(r^{2}\right)=0 .
\end{aligned}
$$

Eq. (25) describes a fourth-fold spiral, while Eq. (26) characterizes a set of centered circles.

Fig. 7 demonstrates a shape of these curves. Transition through these curves corresponds to the field phase leap by $\pi$. The points of intersection of the ring and spiral lines in the figure are doubly degenerate, similarly to that occurring at the centered point. They have nothing to do with the optical vortices. The transition trough the point is not accompanied by the $\pi$-phase leap. The relief of these dislocation lines is well traced by colour-tinted lines in Fig. 6.

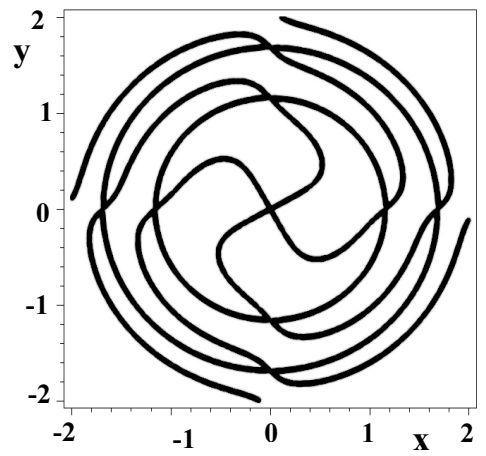

Fig. 7. Spiral and ring wave front dislocations.

Consequently, the lines represent a ring and spiral wave front dislocations [16]. As we have shown in the Appendix, the geometric Pancharatnam phase $[32,33]$ acquired by the beam transmitted through the binary system of chiral crystals plays a major role in producing these dislocations. The socalled transverse geometric phase vanishes along the spiral lines: $\Gamma_{\perp}(r, \varphi)=0$.

Notice that the rotation of polarizer axis by the angle $\pi / 2$ turns the black line into the white one. Naturally, by differentiating the intensity $I_{x} \propto\left|D_{x}\right|^{2}$ over the coordinate $\varphi^{\prime}$ and equating the derivative to zero we come to Eqs. (25) and (26) describing the positions of the intensity maxima.

Thus, the Airy' spiral is a result of interference of two vortex beams with the opposite double topological charges and the opposite signs of their geometrical Pancharatnam phases.

One can reveal that the dark spiral-like line appearing in the conoscopic pattern of coherent beam passed through the binary system of chiral and purely anisotropic crystals is not a spiral wave dislocation. It is produced by a chain of optical vortices, outlining the minimum of the beam intensity in the form of spiral-like line.

Getting finished with the Section, we revert again to a single chiral crystal. We wish to turn to Ref [36] where the authors state they have observed a two-fold Airy' spiral in the beam transmitted through a single chiral crystal. Namely, the linearly polarized light then passed through a chiral crystal and a quarter-wave phase retarder. In fact, their experiment looks like the one mentioned above, involving the binary system of both chiral and anisotropic crystals. Thus, the authors could not observe the Airy' spiral because their experimental set-up did not allow shaping the wave front with a peculiar distribution of the geometrical phase. 


\section{Conclusions}

We have developed a matrix approach for describing propagation of paraxial LaguerreGaussian beams through the system of chiral crystals. We have shown that the circularly polarized singular beams passing such the system carries centered optical vortices with double topological charge. Besides, these beams turn out to acquire the geometrical phase, whose sign is defined by the circulation of the initial beam polarization and the crystal chirality. In order to elucidate the geometrical phase in the beam cross-section, we have employed a superposition of two circularly polarized beams transmitting through the binary system of chiral crystals with the opposite signs of their chirality. Having passed through the system, the beams become superposed. The interference process allows compensation of any deviations of the dynamic phases, while the geometrical phases play a major part in producing the resulting pattern. We have revealed that a geometric set of points, where the geometrical Pancharatnam phase vanishes, forms a four-fold spiral of zero intensity. Transmission through the spiral gives rise in a leap of the beam phase by $\pi$. It means that the spiral represents a pure edge dislocation. In crystallography this singular line is referred to as the Airy' spiral. We have also shown that the spiral-like dark curves in the beam passed through the binary system of chiral and anisotropic crystals are not spiral edge wave front dislocations. They are formed by a chain of optical vortices.

\section{Acknowledgements}

The authors thank R. Vlokh (Institute of Physical Optics, Lviv, Ukraine) and A. Kiselev (Institute of Applied Mathematics, St. Petersburg, Russia) for fruitful discussions and also Yu. Egorov (Taurida National University, Simferopol, Ukraine) for the help in experiments. The stimulus for the paper has been reiterated discussions about crystal-generated optical vortices with Yu. Kivshar and A. Desyatnikov (Australian National University, Canberra).

\section{Appendix: The geometrical phase}

If a wave field is subjected to a series of successive transformations that return the field to the initial state, the wave function of the field acquires an additional phase factor. It means that the wave field transmitting through a complex system accumulates not only a dynamic phase but also a peculiar nonintegrated phase that has come to be called a geometrical phase [37]. Indeed, one can characterize the wave in some space of the wave states. As a rule, the state transformations are accompanied with moving the representative point of the field along some space trajectory. If the wave field returns to its initial state, the trajectory gets closed. For closed trajectories, the phase access or the geometrical phase are proportional to a solid angle formed by a portion of the surface cut out by the closed curve in the state space (see Fig. Al). Obviously, the geometrical phase is not zero or $2 \pi$ if the trajectory is not a plane curve. A well-known type of the geometrical phase is associated with a movement of wave along a spiral trajectory. It is the so-called 


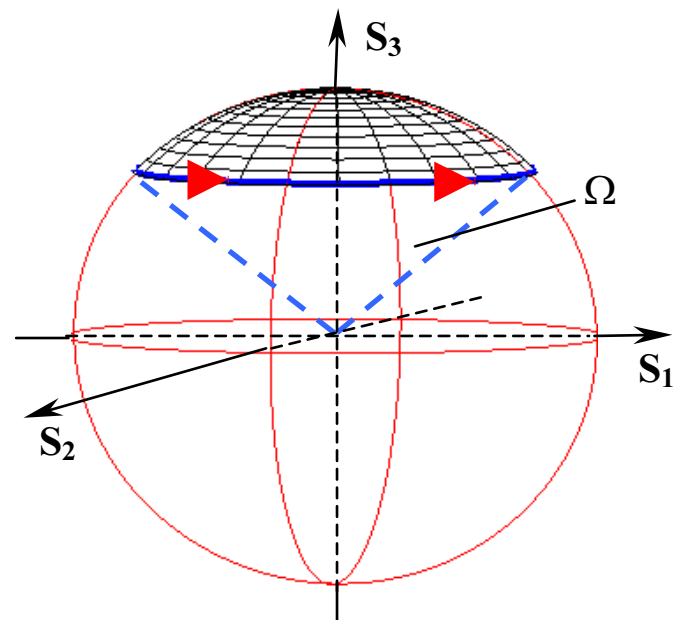

Fig. A1. A trajectory of polarization states on the Poincare sphere caused by a variation of the azimuthal coordinate $\varphi$, plotted in accordance with Eq. (A2) for $\Delta n_{L}=0.02, \Delta n_{C}=1.5 \cdot 10^{-5}$, $d=1 \mathrm{~cm}$ and $r=2 \cdot 10^{-4} \mathrm{~m}$.
Rytov-Vladimirsky-Berry phase [37]. This phase is responsible for rotation of the linear polarization.

The second type of the geometrical phase is associated with a cyclic transformation of the wave polarization state, the socalled Pancharatnam phase [38]. A polarization state of the wave field is characterized by a point lying on the Poincare sphere. The coordinates of the point on the sphere with the unit radius are the Stokes parameters [23]. A closed trajectory cuts out a portion of the sphere surface with the solid angle $\Omega$ and the magnitude of the Pancharatnam phase $\Gamma$ is then equal to $\Gamma=\Omega / 2$ (see Fig. Al).

The Pancharatnam phase can be conventionally divided into the longitudinal $\left(\Gamma_{||}\right)$ and transverse $\left(\Gamma_{\perp}\right)$ geometric phases. For example, a plane wave transmitting through a stack of birefringent plates, whose crystallographic axes are rotated by some angle with respect to each other, experiences a cyclic transformation of its polarization state. Thus, the geometric phase (or, better to say, the longitudinal geometric phase $\Gamma_{||}$) entails variations of the longitudinal coordinate $z$ [32]. However, if a divergent beam of rays propagates along such the stack, there appears a field of nonuniformly distributed polarization states in the beam cross-section at the entrance end of the stack. The polarization states occupy some region on the Poincare sphere. In general, we have infinite number of versions for moving along closed trajectories. As for the physical aspect, moving along a closed trajectory in a curved space is associated with a time matching of events in local reference frames, accompanied by a parallel transport of the vector along the closed contour [39]. A choice of the contour shape is defined by a particular physical task. For example, the contour may be chosen in the form of a curve that encircles the beam axis or a contour lying along some peculiar line etc., so that the closed curve on the Poincare sphere (consequently, the transverse geometrical phase $\Gamma_{\perp}$ ) is a result of variation of both the radial $(r)$ and azimuthal $(\varphi)$ coordinates [33].

In our case related to the propagation of Gaussian beams along a binary system of chiral crystals, we will look for variations of the transverse Pancharatnam phase $\Gamma_{\perp}$. Let us write the Stokes parameters [29] in terms of the electric induction vector D : 


$$
\begin{gathered}
S_{0}=\frac{1}{2}\left(D_{+} D_{+}^{*}+D_{-}^{*} D_{-}\right), \quad S_{1}=\frac{1}{2}\left(D_{+} D_{-}^{*}-D_{+}^{*} D_{-}\right), \\
S_{2}=\frac{i}{2}\left(D_{-} D_{+}^{*}-D_{-}^{*} D_{+}\right), \quad S_{3}=\frac{1}{2}\left(D_{+} D_{+}^{*}-D_{-}^{*} D_{-}\right) .
\end{gathered}
$$

Using Eq. (22) and the notation $\bar{S}_{j}=\frac{S_{j}}{S_{0}}, j=1,2,3$ we come to the expressions

$$
\begin{gathered}
S_{1}=4 F(r) W_{1}(r, \varphi), \quad S_{2}=4 F(r) W_{2}(r, \varphi), \\
S_{3}=F(r)^{2}-4 \cos ^{2} \alpha \sin ^{2} \Lambda^{\prime}\left(\cos ^{2} \Lambda^{\prime}+\sin ^{2} \alpha \sin ^{2} \Lambda^{\prime}\right),
\end{gathered}
$$

with

$$
\begin{gathered}
F(r)=\cos ^{2} \Lambda^{\prime}-\cos 2 \alpha \sin ^{2} \Lambda^{\prime}, W_{1}(r, \varphi)=\cos \Lambda^{\prime} \sin 2 \varphi+\sin \alpha \sin \Lambda^{\prime} \cos 2 \varphi, \\
W_{2}(r, \varphi)=\cos \Lambda^{\prime} \cos 2 \varphi-\sin \alpha \sin \Lambda^{\prime} \sin 2 \varphi .
\end{gathered}
$$

The geometric Pancharatnam phase $\Gamma_{\perp}$ vanishes provided that the representative point moves along the major meridians and the equator [38]. It can take place if either $S_{1}=0$ or $S_{2}=0$. In particular, we find that $\Gamma_{\perp}(r, \varphi)=0$ under the conditions

$$
\cos \Lambda^{\prime} \sin 2 \varphi+\sin \Lambda^{\prime} \cos 2 \varphi=0
$$

and

$$
\cos \Lambda^{\prime} \cos 2 \varphi-\sin \Lambda^{\prime} \sin 2 \varphi=0 .
$$

Both the expressions represent spirals in the coordinates $(r, \varphi)$. In fact, the expression given by Eq. (A4) coincides with Eq. (25) written for the four-fold Airy' spiral.

\section{References}

1. Soskin M, and Vasnetsov M, 2001. Singular optics. Progress in Optics 42: 219-276.

2. Abramochkin E G and Volostnikov V G, 2004. Spiral light beams. Uspekhi Fiz. Nauk. 174: 1273-1300.

3. Kasak NS, Khio NA and Ryzhevich AA, 1999. Generation of Bessel light beams under the condition of internal conical refraction. Quant. Electr. 29: 1020-1024.

4. Volyar A, Fadeyeva T and Egorov Yu, 2002. Vector singularities of Gaussian beams in uniaxial crystals: Optical vortex generation. Techn. Phys. Lett. 28: 70-77.

5. Cincotti G, Ciattoni A and Sapia C, 2003. Radially and azimuthally polarized vortices in uniaxial crystals. Opt. Commun. 220: 33-40.

6. Volyar A and Fadeyeva T, 2003. Generation of singular beams in uniaxial crystals, Opt. Spektrosk. 94: 264-274.

7. Ciattoni A, Cincotti $\mathrm{G}$ and Palma C, 2003. Circular polarized beams and vortex generation in uniaxial media. Journ.Opt.Soc.Am. A 20: 163-171.

8. Vlokh R, Volyar A, Mys O and Krupych O, 2003. Appearing of the vortex at the conical refraction. Example of $\mathrm{NaNO}_{2}$ and $\mathrm{YFeO}_{3}$ crystals. Ukr J.Phys.Opt. 4: 90-93. 
9. Berry M, and Dennis M, 2003. The optical singularities of birefringent dichroic chiral crystals. Proc. Roy. Soc. Lond. A. 459: 1261-1292.

10. Berry M, 2004. Conical diffraction asymptotics: fine structure of Poggendorff rings and axial spike. J. Opt: Pure and Appl. Opt. 6: 289-300.

11. Egorov Yu, Fadeyeva T and Volyar A, 2004. The fine structure of singular beams in crystals: colours and polarization. J. Opt: Pure and Appl. Opt. 6: S217-S228.

12. Novlitsky A and Barkovsky L, 2005. Vector Bessel beams in bianisotropic media. J. Opt: Pure and Appl. Opt. 7: 550-557.

13. Berry M, Jeffrey M and Mansuripur M, 2005. Orbital and spin angular momentum in conical diffraction. J. Opt: Pure and Appl. Opt. 7: 685-690.

14. Berry M, 2005. The optical singularities of bianisotropic crystals. Proc. R. Soc. A. 461: 2071-2098.

15. Plossmann F, Schwarz U, Maier M and Dennis M, 2005. Polarization singularities from unfolding an optical vortex through a birefringent crystal. Phys. Rev. Lett. 95: 253901-1-4

16. Nye J.E. Natural focusing and fine structure of light. Bristol and Philadelphia: Institute of Physics Publishing (1999).

17. Volyar A and Fadeyeva T, 2006. Laguerre-Gaussian beams with complex and real arguments in uniaxial crystals. Optics and Spectroscopy 101: 297-304.

18. Berry $M$ and Jeffrey M, 2006. Chiral conical diffraction. J. Opt: Pure and Appl. Opt. 8: $363-372$.

19. Angelsky O, Maksimyak A, Maksimyak P and Hanson S, 2005. Interference diagnostics of white-light vortices. Opt. Express 13: 8179-8183.

20. Shvedov V, Krohkowski W, Volyar A, Neshev D, Desyatnikov A and Kivshar Yu,. 2005. Focusing and correlation properties of while-light vortices. Opt. Express 13: 7393-7398.

21. Volyar A, Shvedov V, Fadeyeva T, Desyatnikov A, Neshev D, Krollikowski W and Kivshar $\mathrm{Yu}, 2006$. Generation of single-charged optical vortices with an uniaxial crystal. Opt. Express 9: 3724-3729.

22. S. McClain, L. Hillman, R. Chipman, 1993. Polarization ray tracing in anisotropic active media: II Theory and Physics. J. Opt. Soc. Am. A. 10: 2383-2393

23. Born M. and Wolf E. Principles of optics. Oxford, London: Pergamon Press (1968).

24. Yariv A. and Yeh P. Optical waves in crystals. New York: J. Waley and Son Inc (1984).

25. Vlokh O.G., Kobylyansky V.B., Laz'ko L.A., 1974. Light interference in uniaxial gyrotropyc crystals and its application to determination of gyration tensor components. Ukr.Fiz. Zhurn. 19: 1631-1638.

26. Volyar A, Shvedov V and Fadeyeva T, 1999. Topological phase in the nonparaxial Gaussian beam. Techn. Phys. Lett. 25: 891-894. 
27. Courtial $\mathrm{J}$ and Padgett $\mathrm{M}, 2000$. Limit of the orbital angular momentum per unit energy in a light beam that can be focused onto a small particle. Opt. Commun. 173: 269-274.

28. Bekshaev A, Soskin M and Vasnetsov M, 2006. Centrifugal transformation of the transverse structure of freely propagating paraxial light beams. Opt. Lett. 31: 694696.

29. Andrews L. and Phillips R. Mathematical Techniques for Engineers and Scientists. Washington, USA: SPIE Press. (2004).

30. Gori F, 2001. Polarization basis for vortex beams. J. Opt. Soc. Am. A. 18: 16121617.

31. Azzam R. and Bashara N. Ellipsometry and polarized light. Amsterdam, New York: North-Holland publishing company. (1977).

32. Berry M and Klein S, 1995. Geometric phases from stacks of crystal plates. J. Mod. Opt. 43: 165-180.

33. Niv A, Biener G, Kleiner V and Hasman E, 2006. Manipulation of the Pancharatnam phase in vectorial vortices. Opt. Express 14: 4208-4220.

34. Bryngdahl O, 1973. Radial- and circular-fringe interferograms. J. Opt. Soc. Am. 9: 1098-1104.

35. Harris M, Hill CA and Vaughan JM, 1994. Optical helices and spiral interference fringes. Opt. Comm. 106: 161-166.

36. Cedar VA and Glazer AM, 2002. A new view of conoscopic illumination of optically active crystals. Appl. Crystall. 35: 185-190.

37. Berry M. Geometric Phases in Physics. Singapore: World Scientific (1989).

38. Pancharatnam S, 1956. Generalized theory of interference and its applications. Part I. Coherent pencils. Proc. Indian Acad. Sci. A. 44: 247. (reprinted in Geometric Phases in Physics. Singapore: World Scientific (1989)).

39. Landau L.D. and Lifshitz E.M. The Classical Theory of Fields, Fourth Edition: Volume 2 (Course of Theoretical Physics Series), Butterworth-Heinemann (1980). 